\title{
Spectral cues which influence monaural localization in the horizontal plane
}

\author{
KRYSTYNA BELENDIUK and ROBERT A. BUTLER \\ Departments of Surgery (Otolaryngology) and Behavioral Sciences \\ University of Chicago, Chicago, Illinois 60637
}

\begin{abstract}
An extensive series of behavioral tests was carried out to determine what region, or regions, of the sound spectrum were critical for locating sounds monaurally in the horizontal plane. Seven subjects were requested to locate narrow bands of noise centered at different frequencies, combinations of these noise bands, low-pass, high-pass, and broadband noise. As observed in an earlier study, increasing bandwidth did not necessarily lead to improved localization performance until the band became broad, including, for example, all frequencies above $4.0 \mathrm{kHz}$. What seems to be happening is that listeners perceive narrow bands of noise originating from restricted places in the horizontal plane which may differ one from another depending on the frequency composition of the stimulus. In several instances, if two noise bands were presented simultaneously, the resulting stimulus was located with reasonable accuracy provided each component, when presented singly, was perceived as emanating from clearly separate azimuthal positions. If, however, two noise bands, which were perceived to originate from approximately the same azimuthal position when presented singly, were now presented simultaneously, the resulting stimulus still was perceived to originate from the same region of the horizontal plane. This, then, is a case where augmenting the spectral content of the stimulus does not bring about improved performance. We suggest that the expression of judgmental biases in the apparent location of a band of noise may prove useful for understanding why some stimuli of specified width and center frequency are localizable while others are not.
\end{abstract}

The pinna's transformation of the sound field provides spectral cues which are essential for locating sound in the median sagittal plane (MSP). If the pinnae are distorted or their cavities filled with, say, Plasticine, listeners are unable to locate MSP positioned sounds (Bloch, 1893; Gardner \& Gardner, 1973; Jongkees \& Groen, 1946; Roffler \& Butler, 1968a). We now know something about the characteristics of these cues so important for the MSP localization task. First, the stimulus must be complex; localization of sinusoids is based on their frequency and has no relevance to their actual elevation in space. High-frequency tones simply appear to emanate above ones lower in frequency (Pratt, 1930; Roffler \& Butler, 1968b). Also, the presence of higher audio frequencies $->6.0 \mathrm{kHz}-$ is critical for accurate localization in the MSP. Furthermore, if the width of a high-frequency band of noise is increased, listeners perform more proficiently (Butler \& Planert, 1976). This efficient utilization of spectral information to locate sounds in a plane where interaural difference cues are minimal stands as one of the more remarkable phenomena in the field of auditory space perception. ${ }^{1}$

This research formed part of K.B.'s PhD dissertation and was supported in part by the U.S. Public Health Service Grant NS-11154-02.
When head movements are restricted, spectral cues also serve as the basis for monaural localization of sounds in the horizontal plane (HP). It seems reasonable to assume that the same stimulus conditions important for accurate localization of sounds in the MSP should also apply to monaural HP localization. And, for the most part, they do. Again, pinnae distortion or cavity occlusion results in the inability to locate sounds (Butler, 1975). The stimulus must be complex; judgments of the location of sinusoids in the monaural localization task do not correspond to their actual location (Angell \& Fite, 1901; Butler, 1971). Also, the higher audio frequencies $\rightarrow 5.0 \mathrm{kHz}$ -must be included in the sound before a subject can attain maximal accuracy (Belendiuk \& Butler, 1975). But there exists at least one set of stimulus conditions which fails to affect the MSP and the monaural HP localization tasks similarly. To wit: As the bandwidth of an $8.0-\mathrm{kHz}$ centered noise is increased from 2.0 to $6.0 \mathrm{kHz}$, the performance accuracy when locating HP-positioned sounds monaurally may or may not show continued improvement (Butler \& Planert, 1976). ${ }^{2}$ This exception may have wider implications for the mechanisms underlying auditory space perception. If listeners utilize spectral cues on a monaural HP localization task, then increasing the spectral information in the frequency region thought to be important for this task should make for greater accuracy. The reasons for failure to show 
an orderly improvement are not immediately apparent. It may be that frequencies greater than $11.0 \mathrm{kHz}$ are of special significance for monaural localization in the HP and these frequencies were not contained in the bandpass noise bursts alluded to above. On the other hand, different subjects may use different and narrowly circumscribed frequency regions of the sound spectrum for the localization task. By extending such regions only partially, the spectral cues governing localization for a certain listener may become confounded. These considerations are dealt with in the experiment to be reported below.

\section{METHOD}

Subjects were asked to report, via an intercom system, that loudspeaker form which the sounds appeared to emanate. The transducers consisted of five KLH loudspeakers, 4 in. in diameter and housed in wooden cabinets. They were positioned at 345 , $330,315,300$, and $285 \mathrm{deg}$ azimuth. Each was identified by a number, 1 through 5, with " 1 " assigned to the loudspeaker stationed at $345 \mathrm{deg}$ and " 5 " assigned to that stationed at $285 \mathrm{deg}$ azimuth. The test room was sound treated by means of sound-absorbant material on the walls and ceiling and carpeting covering the floor.

Seven normal-hearing persons participated. Their thresholds for frequencies ranging from 0.25 through $8.0 \mathrm{kHz}$ were within $15 \mathrm{~dB}$ re audiometric zero (ISO; see Davis \& Kranz, 1964). We established a monaural listening condition by inserting a Mine Safety Appliance (MSA) ear defender into the right ear canal and then covering the same ear with a MSA muff which was strapped to the head. We measured thresholds to all stimuli used in the experiment, first with only the right ear occluded and again when both ears were occluded. These threshold data enabled us to choose a left-ear sensation level for the test stimuli which was clearly audible and yet below threshold for the right, i.e., the occluded ear. This turned out to be $20 \mathrm{~dB}$. We manipulated loudness by sometimes adding and sometimes subtracting $2 \mathrm{~dB}$ from the signal. Stimulus duration was $30 \mathrm{msec}$; rise-fall time was $10 \mathrm{msec}$.

The frequency composition of the stimuli to be located was chosen to fill the gaps in our knowledge on which frequency regions are critical for proficient monaural localization of HPpositioned sounds. Specifically, we do not know whether lowfrequency sounds- $<4.0 \mathrm{kHz}$ - when added to sounds of higher frequencies improve performance. The Belendiuk and Butler (1975) experiment only investigated the performances as the high-frequency cutoff of a low-pass noise was increased progressively. Nor, as mentioned earlier, do we know whether frequencies $>11.0 \mathrm{kHz}$ contribute toward proficiency in monaural localization in the HP. And lastly, the Butler-Planert (1976) study used just one center frequency, viz., $8.0 \mathrm{kHz}$. To gain a broader perspective on the role of spectral cues in monaural localization, we employed, in a present study, several different center frequencies and bandwidths. In all, 17 different stimulus conditions were established. Our choice of frequency regions reflecting the above considerations is illustrated in Figure 1. Shown here are bandpass noise stimuli centered at three different frequencies $(5.0,7.4$, and $9.8 \mathrm{kHz})$, a low-pass noise whose upper frequency cutoff was $2.5 \mathrm{kHz}$ and a high-pass noise whose lower frequency cutoff was $11.0 \mathrm{kHz}$. The width of the bandpass noise stimuli was $2.4 \mathrm{kHz}$. To generate each bandpass, we used a ring modulator circuit in which the carrier (center frequency) was modulated by a $1.2-\mathrm{kHz}$ low-pass noise. Three filters (SpencerKennedy Laboratories, Model 302), each with a rejection rate of
$18 \mathrm{~dB} /$ octave, were cascaded to filter the output of the noise generator, thereby providing a reasonably restricted band of noise. To generate the $2.5-\mathrm{kHz}$ low-pass noise, a General Radio multifilter, Model 1925, whose rejection rate was approximately $48 \mathrm{~dB}$ /octave, was used to filter a broadband noise stimulus. For the $11.0-\mathrm{kHz}$ high-pass noise, the multifilter was used in conjunction with other filters (SKL and Allison, Model AL2BR) to tailor the broadband noise. The resulting rejection rate was approximately $54 \mathrm{~dB} /$ octave. Also shown in Figure 1 are combinations of the various bands of noise. When bandpass noise stimuli were combined, the carrier frequency and cutoff frequency of the low-pass modulating noise were changed accordingly. So, for example, in combining the $5.0-$ and $7.4-\mathrm{kHz}$ bandpass noise stimuli, the carrier frequency was set at $6.2 \mathrm{kHz}$ and the cutoff frequency for the low-pass modulating noise was set at $2.4 \mathrm{kHz}$. By virtue of increasing the cutoff frequency of the modulating noise, the rejection rate for the resulting bandpass stimulus was somewhat less than for the individual bandpass stimuli. To generate stimuli consisting of a bandpass noise plus either a low- or high-pass noise, the output of the noise generator was split, each component filtered according to the experimental plan, then delivered to a mixer whose output was transmitted to the desired loudspeaker. The combination of $5.0-\mathrm{kHz}$ centered noise band and $9.8-\mathrm{kHz}$ centered noise band required that the latter be filtered by the multifilter as our ring modulator circuit was occupied in fabricating the former stimulus. This substitution approximated, but did not match, the $9.8-\mathrm{kHz}$ centered noise when structured by means of the ring modulator circuit. The spectra for the narrow bands of noise, the low- and high-pass noises, were analyzed and displayed by a Hewlett-Packard Spectrum Analyzer (Model 3480A) and a X-Y recorder (Model 7035B), respectively.

The subjects were given 100 localization trials on each of the 17 stimulus conditions. The presentation order of the various stimulus conditions was varied among listeners and for any one

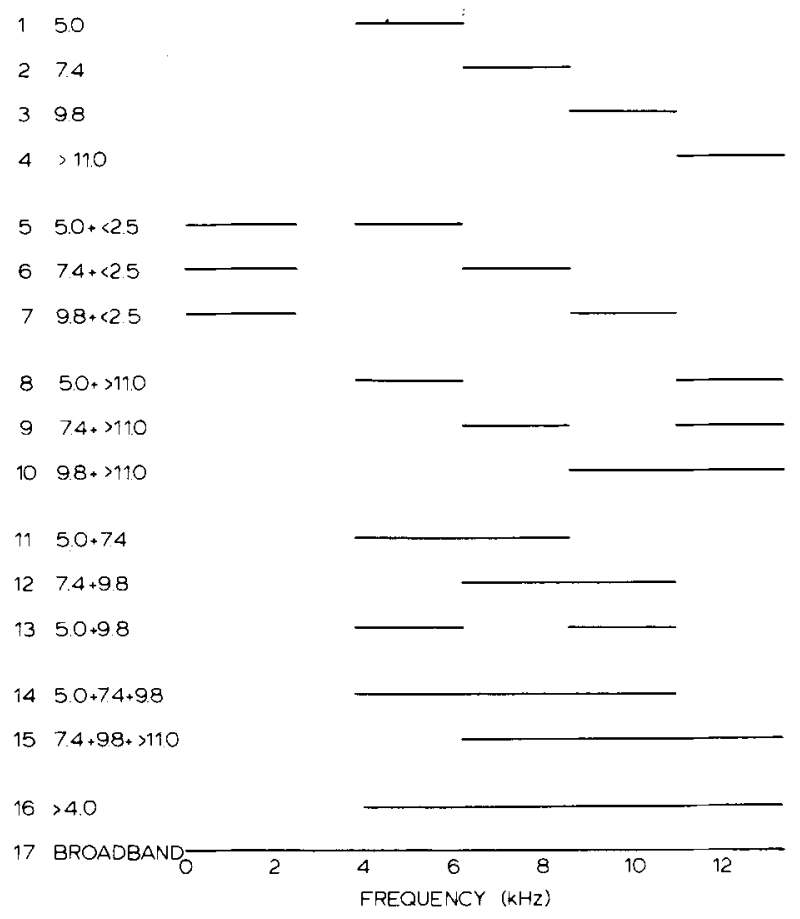

Figure 1. A diagramatic sketch of the various noise stimuli employed. 
condition, the order of loudspeaker presentation was haphazard. Because the subjects were tested repeatedly, we used several different presentation orders for the loudspeakers to reduce the possibility that a subject might learn something about the order in which the transducers were being activated. Localization performances were transformed into error scores. An error score of " 0 " was recorded if the subject correctly identified that loudspeaker from which the sounds emanated. Error scores of "1," " 2 ," "3," or " 4 " were recorded if the subject chose a loudspeaker that was 1 (15 deg), 2 (30 deg), 3 (45 deg), or 4 (60 deg) removed from the one generating the stimuli for that particular trial.

\section{RESULTS}

A chi-square test was performed on each subject's data for each stimulus condition to find out whether the distribution of error scores differed from that expected by chance. In most situations, this criterion would be sufficient for determining whether a listener was locating the sounds at a reasonably accurate level. But, for these series of tests, all error score distributions differed from a chance distribution. Inspection of the data indicated that listeners were exhibiting strong judgmental biases with regard to the region in the horizontal plane from which the sounds appeared to originate. The narrow-band noise stimuli frequently appeared to come from only one or two loudspeakers regardless of their actual position in the HP. Loudspeaker choices for the broader band stimuli were usually distributed among loudspeakers $2,3,4$, and 5 positioned at 330,315 , 300 , and $285 \mathrm{deg}$ azimuth, respectively. Six of the seven subjects rarely chose Loudspeaker 1 (345 deg azimuth) as the generator of the sounds. For purposes of data analysis, we defined a judgmental (location) bias as a distribution of loudspeaker choices which differed significantly $(p<.001)$ from a rectangular distribution as determined by the chisquare test. A rectangular distribution served as the "expected" distribution in the chi-square test, since each loudspeaker was activated an equal number of times during a set of 100 localization trials. To distinguish between an acceptable level (Level 1) and an unacceptable level (Level 2) of localization proficiency, we defined a Level 1 performance as follows: Detecting correctly on at least two-thirds of the trials the direction of a change when the sound was moved by $30 \mathrm{deg}$ re its position on the previous trial. Suppose, for example, that a $7.4-\mathrm{kHz}$ centered noise band originated at $315 \mathrm{deg}$ azimuth on Trial 8 and $285 \mathrm{deg}$ azimuth on Trial 9. If the listener responded by choosing a loudspeaker on Trial 9 which was to the left of that chosen on Trial 8, he was considered as correctly having detected the direction of change in the azimuthal position of the sound source. In 43 of the 45 instances where this criterion was met for the 30-deg change in sound source, it was also met for both 45- and 60-deg changes in the sound source re its location on the previous trial. Our criterion for an acceptable level of proficiencycorrect detection of a directional change when a sound is moved by $30 \mathrm{deg}$ on at least two-thirds of the trials-is a relatively conservative one since in many, if not most, of the test sessions, the subjects frequently chose the same loudspeaker trial after trial. One additional partitioning of the data seemed appropriate: For Level 1 performances, some subjects on a few tests exhibited no judgmental biases. Hence, they were, indeed, locating the sound source accurately. These test results were categorized as Level 1a; all other Level 1 performances were categorized as Level $1 \mathrm{~b}$.

Table 1 provides a detailed summary of the results. Here are listed the total error score for each subject on each stimulus condition as well as the group means of these error scores. A score of 160 would be expected if a listener selected, as the stimulus source, loudspeakers at random. Also listed are the levels of proficiency in locating the various stimuli. Of the 119 tests, performances on 74 of them fell into the Level 2 category, 36 in Level $1 \mathrm{~b}$, and only 9 in Level 1a. Upon perusing this table, several instances of agreement among subjects are apparent. Specifically, localization accuracy was maximal for the group when the stimulus contained all audible frequencies greater than $4.0 \mathrm{kHz}$ (Conditions 16 and 17). Adding frequencies below $4.0 \mathrm{kHz}$ did not, on the average, improve performance (cf. Conditions 16 and 17). In most cases, the subjects did not perform well at locating the bandpass noises centered at 5.0, 7.4, $9.8 \mathrm{kHz}$ (Conditions 1, 2, and 3). Again, adding the low-pass noise to these bandpass stimuli did not improve matters (see total error scores for Conditions 5, 6, and 7). Adding the high-pass noise $(>11.0 \mathrm{kHz})$ to these same stimuli provided a few instances of markedly improved performances (see total error scores for Conditions 8, 9, and 10). Increasing the bandwidth from 4.8 to $7.2 \mathrm{kHz}$ did not always lead to improved (Compare Condition 10 with Condition 15, Condition 11 with Condition 14, and Condition 12 with Condition 14).

In short, there are several instances to be found in Table 1 where adding a band of noise contiguous or noncontiguous in frequency to another noise band of a fixed center frequency failed to reduce total error scores. Yet, there are other cases where total error scores dropped precipitously when the bandwidth was increased. In an attempt to gain insight into the situation, we examined, for each subject, the apparent location of each noise band employed in the study; i.e., we plotted the distribution of loudspeaker choices for each stimulus condition. Our plots-loudspeaker choice as a function of frequency composition of the stimulus-suggested the following rule: The addition of a noise band, centered at 
Table 1

Total Error Score per 100 Trials for Each Subject on Each Stimulus Condition, and Mean Error Scores (M) for the Group

\begin{tabular}{|c|c|c|c|c|c|c|c|c|c|}
\hline \multirow{2}{*}{$\begin{array}{c}\text { Condi- } \\
\text { tion }\end{array}$} & \multirow{2}{*}{$\begin{array}{l}\text { Frequency } \\
\text { Composition }\end{array}$} & \multicolumn{7}{|c|}{ Subject } & \multirow[b]{2}{*}{$\mathbf{M}$} \\
\hline & & 1 & 2 & 3 & 4 & 5 & 6 & 7 & \\
\hline 1 & $5.0 \mathrm{kHz}$ & $119(1 \mathrm{~b})$ & $117(2)$ & $137(2)$ & $105(2)$ & $130(2)$ & $139(2)$ & $140(2)$ & 126.7 \\
\hline 2 & 7.4 & 65 (1b) & $102(1 b)$ & $143(2)$ & $113(2)$ & $170(2)$ & $200(2)$ & $125(2)$ & 131.1 \\
\hline 3 & 9.8 & $140(2)$ & $121(2)$ & $160(2)$ & $108(2)$ & $190(2)$ & $120(2)$ & $130(2)$ & 138.4 \\
\hline 4 & $>11.0$ & $200(2)$ & $139(2)$ & $110(2)$ & 45 (1a) & $198(2)$ & $51(1 \mathrm{~b})$ & $189(2)$ & 133.1 \\
\hline 5 & $5.0+<2.5$ & $127(2)$ & $102(2)$ & $170(2)$ & $111(2)$ & $135(2)$ & $131(2)$ & $140(2)$ & 130.8 \\
\hline 6 & $7.4+<2.5$ & $57(1 \mathrm{~b})$ & $137(2)$ & $148(2)$ & $127(2)$ & $175(2)$ & $200(2)$ & $131(2)$ & 139.3 \\
\hline 7 & $9.8+<2.5$ & $144(2)$ & $139(2)$ & $152(2)$ & $98(2)$ & $182(2)$ & 169 (2) & $139(2)$ & 146.1 \\
\hline 8 & $5.0+>11.0$ & 96 (1b) & 139 (2) & $121(2)$ & $148(2)$ & $142(2)$ & 48 (1b) & $184(2)$ & 125.4 \\
\hline 9 & $7.4+>11.0$ & 59 (1b) & 84 (1b) & $31(1 \mathrm{~b})$ & 32 (1a) & $132(2)$ & $187(2)$ & 141 (2) & 95.1 \\
\hline 10 & $9.8+>11.0$ & $131(2)$ & $55(1 b)$ & $42(1 b)$ & 23 (1a) & $105(2)$ & 54 (1b) & 80 (1b) & 70.0 \\
\hline 11 & $5.0+7.4$ & 32 (1b) & $96(2)$ & $91(2)$ & 87 (1b) & $120(2)$ & $183(2)$ & $109(2)$ & 102.6 \\
\hline 12 & $7.4+9.8$ & 24 (1b) & $144(2)$ & $139(2)$ & $68(1 b)$ & $156(2)$ & $74(1 b)$ & $107(2)$ & 101.7 \\
\hline 13 & $5.0+9.8$ & $113(2)$ & $141(2)$ & $129(2)$ & $72(1 b)$ & $139(2)$ & $108(2)$ & 81 (1b) & 11.9 \\
\hline 14 & $5.0+7.4+9.8$ & 27 (1b) & $96(2)$ & $118(2)$ & $83(1 b)$ & $116(2)$ & $103(2)$ & $104(2)$ & 92.4 \\
\hline 15 & $7.4+9.8+>11.0$ & 5 (1a) & $30(1 b)$ & $155(2)$ & $40(1 b)$ & $72(1 \mathrm{~b})$ & $157(2)$ & 22 (1a) & 68.7 \\
\hline 16 & $>4.0$ & 10 (1a) & 27 (1b) & 49 (1b) & 9 (1a) & $71(1 \mathrm{~b})$ & $74(1 \mathrm{~b})$ & 34 (1b) & 39.1 \\
\hline 17 & $\mathrm{Bb}$ & $17(1 a)$ & $32(1 b)$ & $59(1 b)$ & $3(1 a)$ & $63(1 \mathrm{~b})$ & $66(1 \mathrm{~b})$ & $34(1 b)$ & 39.1 \\
\hline
\end{tabular}

Note-Proficiency levels associated with each error score are placed in parentheses. Bb stands for broadband noise.

one frequency, to another noise band centered at a different frequency will result in improved localization performance if the distribution of loudspeaker choices for each noise band when presented singly differs from one another in a pronounced manner. If, on the other hand, the choice of loudspeaker distribution for each noise band overlaps appreciably, then combining the two noise bands will not result in improved localization performances. Here, then, may be the place in the analysis of the results where the judgmental biases, so prevalent in the data, become informative: They may tell us which constituents of a complex sound contribute toward accuracy in its localization. Some examples drawn to support these statements follow for which Table 1 as well as the relevant figures should be consulted as the description of our results progresses. Take the performances of Subject 1 on Conditions 2, 3, and 12 , where the total error scores were 65,140 , and 24 , respectively. The distributions of loudspeaker choices for the three conditions are shown in Figure $2 \mathrm{a}$. Loudspeaker 2 (330 deg azimuth) was chosen on more than half the trials when Condition 2 $(7.4 \mathrm{kHz}$ centered noise band) was presented. Loudspeaker 4 (300 deg azimuth) was chosen on all the trials when Condition $3(9.8 \mathrm{kHz}$ centered noise band) was presented. These loudspeaker choices, although clearly biased, were biased in a different way. When both noise bands were presented simultaneously (Condition 12), the loudspeaker choices were more evenly distributed among Loudspeakers $2,3,4$, and 5 and this was accompanied by a marked reduction in the total error score. Consider, now, the performance for the same listener when locating the stimuli of Conditions 1,3 , and 13 (Figure $2 \mathrm{~b}$ ). Here the plurality of location responses for the $5.0-\mathrm{kHz}$ centered noise band was directed toward Loudspeaker 4 and all of the localization responses to the $9.8-\mathrm{kHz}$ centered noise band were directed toward this same loudspeaker. Presenting the two bandpass noises simultaneously resulted again in the choice of Loudspeaker 4 on most trials. In brief, increasing the spectral information failed to improve localization accuracy; perhaps each noise band provided the same locale information about the sound source. This phenomenon can be seen again in the data for Subject 2 which is illustrated in Figure 3. The loudspeaker choices for the $9.8-\mathrm{kHz}$ centered noise band differed from that for the 11.0$\mathrm{kHz}$ high-pass noise stimulus. Combining the two stimuli resulted in a more even distribution of loudspeaker choices among the three central loudspeaker positions $(330,315$, and $300 \mathrm{deg}$ azimuth) and a

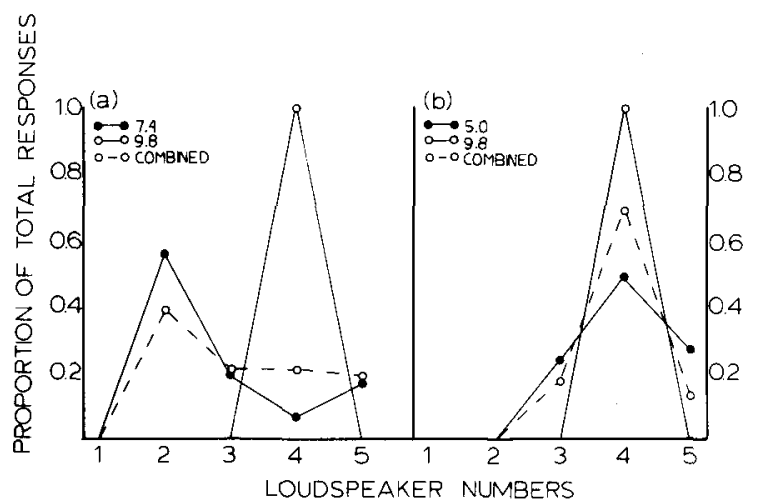

Figure 2. The relative proportion of total location judgments for which each loudspeaker was chosen as the source of the sound. Loudspeakers $1,2,3,4$, and 5 were positioned at 345 , $330,315,300$, and $285 \mathrm{deg}$ azimuth, respectively. Differently constituted noise bands served as the parameter. The data are those of Subject 1. 


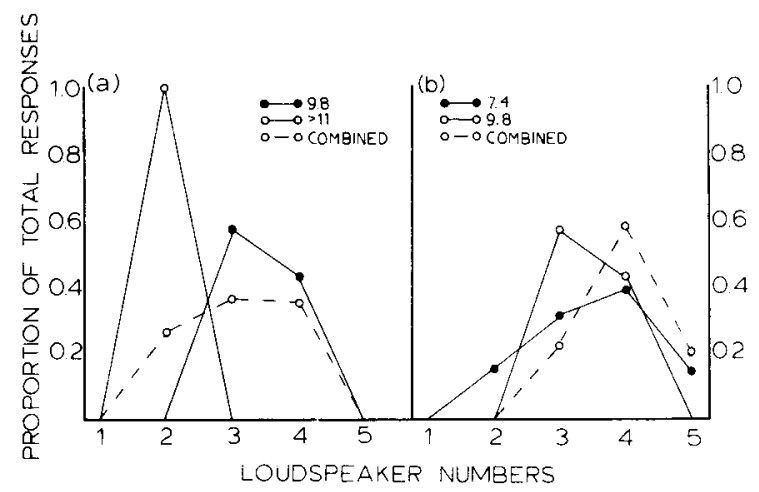

Figure 3. Distribution of loudspeaker choices of Subject 2 for selected noise bands.

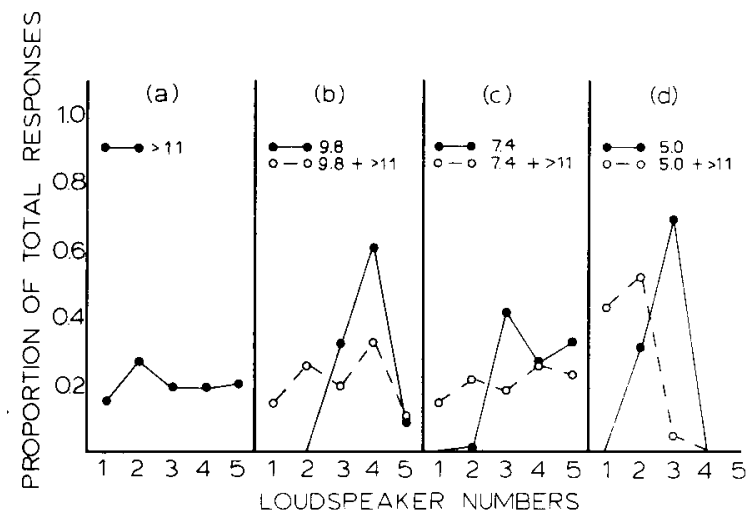

Figure 4. Distribution of loudspeaker choices of Subject 4 for selected noise bands.

reduction in total error scores. On the other hand, the distribution of loudspeaker choices for the 7.4-kHz centered noise band was similar to the noise band centered at $9.8 \mathrm{kHz}$ in that localization judgments were biased toward Loudspeakers 3 and 4 . Presenting the two together failed to improve localization performance. Figure 4 illustrates the localization performances of Subject 4. She was able to locate sounds with a proficiency level of 1a when only the $11.0-\mathrm{kHz}$ high-pass noise was presented. Adding other noise bands to this stimulus either failed to improve or actually disrupted localization performances (see Figures 4b, 4c, and 4d). According to our supposition, all the spectral information essential for locating sounds monaurally in the HP for her should be contained in the $11.0-\mathrm{kHz}$ highpass noise stimulus. Hence, one would expect that different regions of the HP would be represented by narrow bands of noise centered at different frequencies contained in this stimulus. As the listener was still available for further investigation, we conducted a series of special tests on her. Briefly, noise bands, $1.0 \mathrm{kHz}$ wide and centered at $11.0,12.0$, 13.0 , and $14.0 \mathrm{kHz}$ were presented at $20 \mathrm{~dB}$ SL re left (unoccluded) ear. Each noise band was presented
10 times in a haphazard order. The test room and the loudspeaker positions remained the same. Again, the subject reported that loudspeaker from which the sounds came, but unbeknown to her, the sounds always originated from Loudspeaker 3 (315 deg azimuth). Table 2 lists the number of times each loudspeaker was identified as the source of the various narrow bands of noise. Most stimuli centered at 11.0 and $12.0 \mathrm{kHz}$ were perceived as coming from Loudspeaker 4 ; stimuli centered at 13.0 and $14.0 \mathrm{kHz}$ appeared to come from Loudspeakers 1 and 2. Perhaps these differences in judgmental biases for the constituents of an $11.0-\mathrm{kHz}$ high-pass noise stimulus can account for her proficient performance (Level 1a) when locating that high-pass noise during the original test.

Not all cases of differently distributed loudspeaker choices for the individual narrow bands of noise led to improved localization performances when the noise bands were combined, but the data of only one subject (No. 5) clearly refuted our hypothesis. We should point out, however, that this subject did not perform with distinction on any of the stimulus conditions (see Table 1).

\section{DISCUSSION}

In general, proficiency in locating sounds monaurally in the HP improved with increases in the bandwidth of the stimulus. But, as shown by Butler and Planert (1976) and confirmed by the present study, accuracy on this task does not improve in an orderly manner as the bandwidth is augmented. In many cases, increased bandwidth resulted in a decrement in localization performance, and this is the point that most concerns us. What may be happening is that different individuals utilize different and restricted frequency regions of the sound spectrum for locating sounds monaurally. Some examples were presented which pointed to the listener's judgmental biases as providing the cues to the particular frequency regions utilized by different people. If a listener perceives one noise band as originating at one place in the HP which is clearly separated from the perceived location of another noise band, then

Table 2

Distribution of Loudspeaker Choices for Subject 4 as a Function of the Center Frequency of Narrow Bands of Noise

\begin{tabular}{ccrccc}
\hline & \multicolumn{4}{c}{ Loudspeaker } \\
\cline { 2 - 5 } Center & 1 & 2 & 3 & 4 & 5 \\
\hline $11.0 \mathrm{kHz}$ & & & & 7 & 3 \\
$12.0 \mathrm{kHz}$ & & 2 & & 7 & 1 \\
$13.0 \mathrm{kHz}$ & 4 & 6 & & & \\
$14.0 \mathrm{kHz}$ & & 10 & & \\
\hline
\end{tabular}

Note-Each noise band was presented 10 times and always from Loudspeaker 3. 
the two bands, when presented together, should be localizable. The simultaneous presentation of two different noise bands, each perceived when presented alone as coming from the same segment of the HP, will not, when combined, result in improved localization performance. While the data do not support our hypothesis unequivocally, we wish to make the point that the noise bands used in this experiment were not chosen to investigate the role of judgmental biases in the monaural localization of sound in the HP. It so happened that judgmental biases were ubiquitous and they were simply drawn upon in an attempt to explain why in one case increasing the bandwidth resulted in improved localization and why in another case it did not. The data are considered to be of heuristic value, suggesting that a detailed analysis of judgmental biases may lead to a more complete understanding of the spectral basis of monaural localization.

\section{REFERENCES}

ANGELl, J. R., \& Fite, W. The monaural localization of sound. Psychological Review, 1901, 8, 225-246.

Belendiuk, K., \& Butler, R. A. Monaural localization of lowpass noise bands in the horizontal plane. Journal of the Acoustical Society of America, 1975, 58, 701-705.

BLOCH, E. Das binaurale Horen. Zeitschrift für Ohrenheilkunde, $1893,24,25-85$.

Butler, R. A. The monaural localization of tonal stimuli. Perception \& Psychophysics, 1971, 9, 99-101.

Butler, R. A. The influence of the external and middle ear on auditory discriminations. In W. D. Keidel \& W. D. Neff (Eds.),
Handbook of sensory physiology (Vol. 5, Part 2). Berlin: Springer-Verlag, 1975.

Butler, R. A., \& Planert, N. The influence of stimulus bandwidth on localization of sound in space. Perception \& Psychophysics, 1976, 19, 103-108.

Davis, H., \& KRANz, F. W. The international standard reference zero for pure-tone audiometers and its relation to the evaluation of impairment of hearing. Journal of Speech and Hearing Research, 1964, 7, 7-16.

Gardner, M. B., \& Gardner, R. S. Problem of localization in the median plane: Effect of pinnae cavity occlusion. Journal of the Acoustical Society of America, 1973, 53, 400-408.

JongkeEs, L. B. W.. \& Groen, J. J. On directional hearing. Journal of Laryngology and Otology, 1946, 61, 494-504.

Pratt, C. C. The spatial character of high and low tones. Journal of Experimental Psychology, 1930, 13, 278-285.

Roffler, S. K., \& BUTLER, R. A. Factors that influence the localization of sound in vertical plane. Journal of the Acoustical Society of America, 1968, 43, 1255-1259. (a)

Roffler, S. K., \& ButLer, R. A. Localization of tonal stimuli in the vertical plane. Joumal of the Acoustical Society of America, $1968,43,1260-1266$. (b)

Searle, C. L., Braida, L. D., Cuddy, D. R., \& Davis, M. F. Binaural pinna disparity: Another auditory localization cue. Journal of the Acoustical Society of America, 1975, 57. 448-455.

\section{NOTES}

1. Although spectral disparities between ears exist (Searle, Braida, Cuddy, \& Davis, 1975), those interaural differences in time and intensity so important for horizontal plane localization are inoperative in the median sagittal plane.

2. A Friedman chi-square test recently performed on these data indicated that differences in localization accuracy among bandwidths of $2.0,3.0,4.0,5.0$, and $6.0 \mathrm{kHz}$ failed to reach significance at the .05 confidence level. 